\title{
Slinky Inflation
}

\author{
Gabriela Barenboim \\ Departament de Física Teòrica, Universitat de València, \\ Carrer Dr. Moliner 50, E-46100 Burjassot (València), Spain
}

\author{
Joseph Lykken \\ Theoretical Physics Department, \\ Fermi National Accelerator Laboratory, \\ P.O. Box 500, Batavia, Illinois 60510 USA
}

\begin{abstract}
We present a new approach to quintessential inflation, in which both dark energy and inflation are explained by the evolution of a single scalar field. We start from a simple scalar potential with both oscillatory and exponential behavior. We employ the conventional reheating mechanism of new inflation, in which the scalar decays to light fermions with a decay width that is proportional to the scalar mass. Because our scalar mass is proportional to the Hubble rate, this gives adequate reheating at early times while shutting off at late times to preserve quintessence and satisfy nucleosynthesis constraints.

We discuss a simple model which solves the horizon, flatness, and "why now" problems. Without any additional tuning of parameters, this model satisfies all constraints from CMB, large scale structure, and nucleosynthesis. The predictions for the inflationary spectral indices are $n_{S}=n_{T}=1$. In this model we are currently beginning the third cosmic epoch of accelerated expansion.
\end{abstract}

PACS numbers: 98.80.Cq, 95.35.+d, 98.70.Vc

FERMILAB-PUB-05-039-T

If we assume the correctness of the standard Friedmann equation evolution, the existence of dark energy engenders two profound dilemmas. The first is the cosmological constant problem, and the second is the "why now?" problem. Quintessence models attempt to address the second problem by introducing a very weakly coupled scalar field whose potential and/or kinetic function have special properties. One of the most successful approaches to quintessence [1, 2] is to combine tracking with an oscillating behavior in the quintessence potential. In such models the equation of state parameter $w(z)$ has a periodic component, leading to occasional periods of accelerated expansion during epochs where $w(z) \simeq-1$.

It is natural in this context to ask whether the quintessence scalar could replace the inflaton. The idea of quintessential inflation has been examined by a number of authors $[3]-10]$. The straightforward approach is to cobble together a scalar potential which has both a flat, large vev portion (for inflation) and a flat, small vev portion (for quintessence). These features are connected by a steep step which corresponds to a period of cosmic kination. As discussed in 7, 10, such models suffer from generic problems. First, they require significant ad hoc tuning to simultaneously produce the features of inflation and quintessence. Second, they require a "sterile" inflaton, in order to avoid the decay of the putative quintessence scalar at the end of inflation. This in turn requires new mechanisms for reheating, such as gravitational de Sitter phase particle production, leading to difficulties in satisfying the constraints of CMB anisotropies and of primordial nucleosynthesis.

Our approach to quintessential inflation is to take ad-

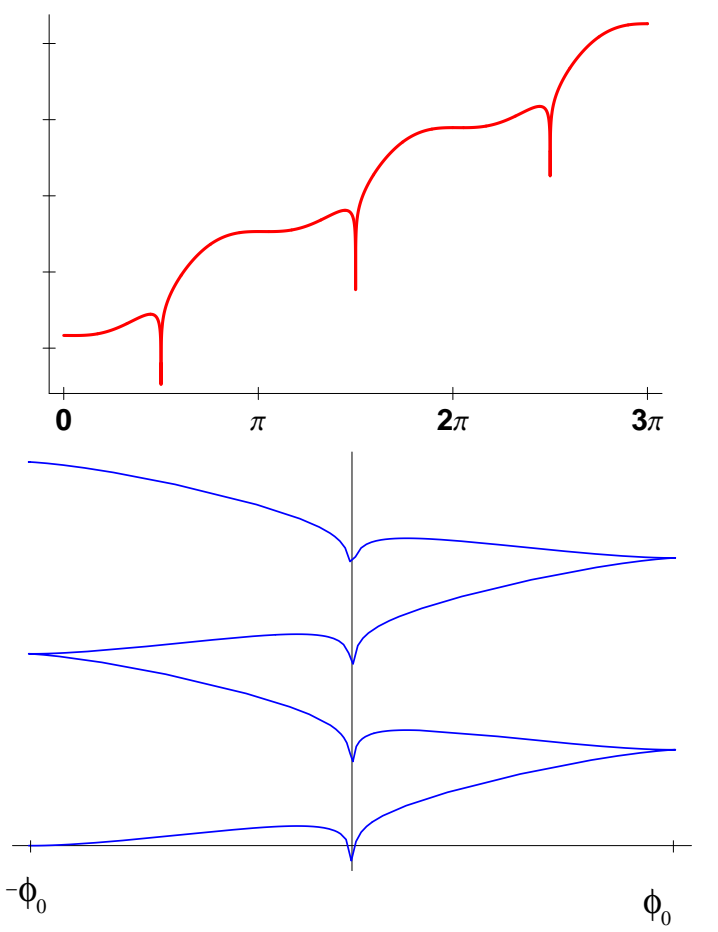

FIG. 1: The upper (lower) panel shows the scalar potential $V(\theta)(V(\phi))$ for $b=1$, plotted on a logarithmic scale.

vantage of the tracking and oscillatory potential features that work so well in addressing the "why now" problem of quintessence alone. We will describe a model with a simple scalar lagrangian with exponential and oscillatory features. The model uses the conventional reheating mechanism of new inflation [11], in which the scalar 
decays to light fermions with a decay width that is proportional to the scalar mass. We show that the scalar mass is proportional to the Hubble rate. As a result, the model has adequate reheating at early times while naturally shutting off at later times.

We present a successful model with only three adjustable parameters. One parameter controls the period between inflationary epochs, a second controls the overall decay width, and the third parametrizes our ignorance about the relative fraction of matter versus radiation produced by reheating. These three parameters are adjusted to produce sufficient inflation along with the correct fractions $\Omega_{r} / \Omega_{\Lambda}, \Omega_{m} / \Omega_{\Lambda}$ of radiation, matter, and dark energy, as measured today. Having thus fixed the model we find that we automatically satisfy all constraints of primordial nucleosynthesis, CMB, and large-scale structure.

\section{AN OSCILLATORY POTENTIAL}

We start with a simple model with a single real scalar quintessence field $\theta$. The action is

$$
\int d^{4} x \sqrt{-g}\left[\frac{1}{2} f(\theta) g^{\mu \nu} \partial_{\mu} \theta \partial_{\nu} \theta-V(\theta)\right],
$$

where the kinetic function $f(\theta)$ and potential $V(\theta)$ are given by:

$$
\begin{aligned}
f(\theta) & =\frac{3 M_{\mathrm{P}}^{2}}{\pi b^{2}} \sin ^{2} \theta \\
V(\theta) & =\rho_{0} \cos ^{2} \theta \exp \left[\frac{3}{b}(2 \theta-\sin 2 \theta)\right],
\end{aligned}
$$

where $M_{\mathrm{P}}$ is the Planck mass: $1.22 \times 10^{19} \mathrm{GeV} ; \rho_{0}$ is the dark energy density observed today: $\simeq\left(10^{-4} \mathrm{eV}\right)^{4} ; b$ is a dimensionless parameter which controls the periodic behavior. A canonical kinetic term can be restored via a field redefinition $\theta(x) \rightarrow \phi(x)$, where

$$
\phi(x)=\phi_{0} \cos \theta
$$

with $\phi_{0} \equiv \sqrt{3 M_{\mathrm{P}}^{2} / \pi b^{2}}$.

In the approximation where we ignore the energy density of radiation and matter, and where the only friction is from the metric expansion, the evolution of the model can be solved analytically. Energy conservation requires:

$$
\dot{\rho}_{\theta}=-3 H(1+w) \rho_{\theta},
$$

where $H$ is the Hubble rate, $w$ is the equation of state parameter, and $\rho_{\theta}$ is the dark energy density. The solution to this equation as a function of the scale factor $a(t)$ is:

$$
\rho_{\theta}(a)=\rho_{0} \exp \left[-3 \int_{1}^{a} \frac{d a}{a}(1+w(a))\right],
$$

where $\rho_{0}$ is the dark energy density at $a=1$ (today).
We also know that

$$
V(\theta)=\frac{1}{2}(1-w) \rho_{\theta} .
$$

Making the Ansatz

$$
w(a)=-\cos 2 \theta(a)
$$

one immediately gets a solution to the (flat) Friedmann equation combined with the relations (6.7):

$$
\begin{aligned}
\theta(a) & =-\frac{b}{2} \ln a \\
w(a) & =-\cos [b \ln a] .
\end{aligned}
$$

The expectation value of the quintessence field $\theta$ evolves logarithmically with scale factor from a positive initial value to zero today. The potential $V(\theta)$ has the "Slinky" form ${ }^{1}$ shown in Figure 1 Accelerated expansion corresponds to epochs (such as today) where $\theta$ is evolving through one of the flat "steps" of the potential. From (2) we see that the kinetic function is simultaneously suppressed in this epochs, slowing the roll of the scalar field evolution. Note also that our potential $V(\theta) \rightarrow 0$ as $\theta \rightarrow-\infty$, which corresponds to $t \rightarrow \infty$; this is as desired for a quintessence model.

We can understand the same behavior by looking at the "canonical" scalar $\phi$ in (4). The potential $V(\phi)$ resembles a series of descending ramps, with the value of $\phi$ bounded between $-\phi_{0}$ and $\phi_{0}$. Shifted around a generic vev $\langle\phi\rangle$, the scalar has an effective mass-squared given by

$$
\frac{m_{\phi}^{2}}{H^{2}}=\frac{b^{2}}{4}+\frac{3}{2} b\left[\frac{\cos ^{3} \theta}{\sin \theta}-2 \sin ^{2} 2 \theta\right]+\frac{9}{2} \sin ^{2} 2 \theta,
$$

where $H$ is the Hubble rate. Instead of a suppression of the kinetic function when $\theta$ is a multiple of $\pi$, the scalar $\phi$ has an effective mass that diverges when $\phi= \pm \phi_{0}$, i.e., at each junction of the descending ramps.

The equation of state parameter $w(a)$ has the same periodic form assumed in two recent phenomenological analyses [12, 13]. The analysis in 12] showed that, for $b=1, w(a)$ as given in (10) is consistent with all current data from observations of the CMB, Type IA supernovae, and large scale structure. It follows a fortiori that our model with any choice of $b$ less than one also agrees with this data.

To complete the model, we will assume that the quintessence field $\phi$ has a weak perturbative coupling to light fermions. This is the standard reheating mechanism of new inflation 11]. To avoid the strong constraints on

\footnotetext{
1 A Slinky $^{T M}$ is a large spring with a very weak spring constant. A Slinky will "walk" down a staircase, producing in time-lapse a pattern resembling the top panel of Figure 1
} 
long-range forces mediated by quintessence scalars [14], it is simplest to imagine that our scalar only has a direct coupling to a sterile neutrino. This is sufficient to hide the quintessence force from Standard Model nonsinglet particles [15], while still allowing the generation of a radiative thermal bath of Standard Model particles from quintessence decay.

With this assumption for reheating the evolution equations for quintessence, radiation, and matter become:

$$
\begin{aligned}
\dot{\rho}_{\theta} & =-3 H(1+w) \rho_{\theta}-k_{0} m_{\phi}(1+w) \rho_{\theta} \\
\dot{\rho}_{r} & =-4 H(1+w) \rho_{r}+\left(1-f_{m}\right) k_{0} m_{\phi}(1+w) \rho_{\theta} \\
\dot{\rho}_{m} & =-3 H(1+w) \rho_{m}+f_{m} k_{0} m_{\phi}(1+w) \rho_{\theta}
\end{aligned}
$$

where $k_{0}$ and $f_{m}$ are small dimensionless constants. From (11) we see that, as long as $\theta$ is not near a multiple of $\pi / 2$, it is a reasonable approximation to make the replacement

$$
k_{0} m_{\phi} \rightarrow k H
$$

where $k$ is another small dimensionless parameter. This replacement decouples the $\theta$ evolution equation from the Friedmann equation, giving an immediate analytic solution:

$$
\rho_{\theta}(a)=\rho_{0} \exp \left[\frac{1}{b}(3+k)(2 \theta-\sin 2 \theta)\right],
$$

where $\theta(a)$ and $w(a)$ are still given by (9)-(10).

We have used this approximation in the solutions quoted below. Clearly this approximation overestimates the reheating effect for epochs where $\theta$ is close to an odd multiple of $\pi / 2$, however this is a small effect since these are the epochs of maximum radiation or matter domination. The approximation also breaks down in the epochs of maximum inflation, i.e. when $\sin \theta \rightarrow 0$. However when $\sin \theta \rightarrow 0$ we also expect strong-coupling physics to enter in a full theory, providing a cutoff for the naive vanishing of the kinetic function of $\theta$. Since we cannot compute this effect without a full Planck-scale theory, we may as well stay with the approximation (13).

\section{RESULTS}

Figure 2 and Table 1 show the results obtained from our model with $b=1 / 7, k=0.06$, and $f_{m}=10^{-11}$. Shown are the relative energy density fractions in dark energy, radiation, and matter, as a function of $\log a$. We have chosen to integrate the evolution equations starting from $a=10^{-42}$, which in our model corresponds to a temperature of slightly less than $10^{16} \mathrm{GeV}$, and an initial comoving Hubble radius of about 100 Planck lengths. For simplicity we have also chosen the value of $w(a)$ now to be exactly -1 . Neither of these choices corresponds to a necessary tuning.

Our three adjustable parameters have been chosen such that the values of $\Omega_{r} / \Omega_{\Lambda}, \Omega_{m} / \Omega_{\Lambda}$ come out to their

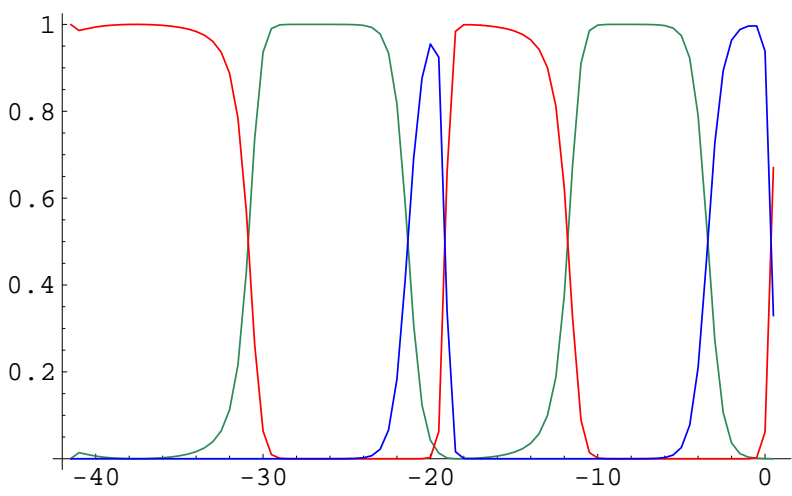

\begin{tabular}{|c|c|c|c|c|c|c|}
\hline $\log _{10} a$ & -42 & -40 & -38 & -36 & -34 & -32 \\
\hline$w(a)$ & -0.32 & -0.83 & -1 & -0.75 & -0.19 & 0.45 \\
\hline$\Omega_{\Lambda}$ & 1 & 0.997 & 0.99998 & 0.996 & 0.975 & 0.78 \\
\hline$\Omega_{r}$ & 0 & 0.003 & $210^{-5}$ & \begin{tabular}{|l|}
0.004 \\
\end{tabular} & \begin{tabular}{|l|}
0.025 \\
\end{tabular} & \begin{tabular}{|l}
0.22 \\
\end{tabular} \\
\hline$\Omega_{m}$ & 0 & $510^{-14}$ & $310^{-16}$ & $510^{-14}$ & $510^{-13}$ & $210^{-11}$ \\
\hline $\log _{10} a$ & -30 & -28 & \begin{tabular}{|l|}
-26 \\
\end{tabular} & \begin{tabular}{|l|}
-24 \\
\end{tabular} & -22 & -20 \\
\hline$w(a)$ & 0.90 & 0.98 & 0.64 & 0.04 & -0.58 & -0.96 \\
\hline$\Omega_{\Lambda}$ & 0.01 & $810^{-7}$ & $410^{-10}$ & $210^{-10}$ & $510^{-7}$ & 0.062 \\
\hline$\overline{\Omega_{r}}$ & 0.99 & 0.999999 & 0.99993 & \begin{tabular}{|l|}
0.993 \\
\end{tabular} & 0.59 & 0.013 \\
\hline$\Omega_{m}$ & $710^{-9}$ & $710^{-7}$ & $710^{-5}$ & 0.007 & 0.41 & 0.925 \\
\hline $\log _{10} a$ & -18 & -16 & -14 & -12 & -10 & -8 \\
\hline$w(a)$ & -0.94 & -0.52 & 0.11 & 0.69 & 0.99 & 0.87 \\
\hline$\Omega_{\Lambda}$ & 0.9992 & 0.99 & 0.94 & 0.32 & 0.0002 & $1110^{-8}$ \\
\hline$\Omega_{r}$ & 0.0008 & 0.01 & 0.06 & 0.68 & 0.9998 & 0.9999 \\
\hline$\Omega_{m}$ & $210^{-5}$ & $610^{-10}$ & $410^{-11}$ & $610^{-9}$ & $810^{-7}$ & $810^{-5}$ \\
\hline $\log _{10} a$ & -6 & -4 & \begin{tabular}{|l|}
-2 \\
\end{tabular} & $\begin{array}{l}0 \\
\end{array}$ & \begin{tabular}{|l|}
2 \\
\end{tabular} & 4 \\
\hline$w(a)$ & 0.39 & -0.24 & -0.79 & -1 & -0.79 & -0.25 \\
\hline$\Omega_{\Lambda}$ & $110^{-10}$ & $210^{-9}$ & $510^{-6}$ & 0.67 & 0.997 & 0.98 \\
\hline$\Omega_{r}$ & 0.992 & 0.54 & 0.01 & 0.00005 & 0.003 & 0.02 \\
\hline$\Omega_{m}$ & 0.008 & 0.46 & 0.99 & 0.33 & $110^{-6}$ & $810^{-10}$ \\
\hline
\end{tabular}

FIG. 2: History of a slinky universe. Shown are the relative energy density fractions in radiation (green), matter (blue), and dark energy (red), as a function of the logarithm of the scale factor $a(t)$.

TABLE I: The relative density fractions of dark energy, radiation, and matter, as a function of the scale factor. The future projections are only valid if the quintessence scalar is still decaying.

measured values at $a=1$, and such that we have sufficient inflation. The latter is checked by computing the ratio of the fully inflated size of the intitial comoving Hubble radius to the current comoving Hubble radius. This ratio is about 3 in our model, indicating that the total amount of inflation is indeed enough to solve the horizon problem. The flatness problem is solved because the total $\Omega_{r}+\Omega_{m}+\Omega_{\Lambda}=1$ within errors.

From Figure (2) we see that we are currently beginning 
the third epoch of accelerated expansion. The first epoch of inflation accumulated about $18 e$-foldings. Quantum fluctuations during this epoch produced the spatial inhomogeneities responsible for large scale structure and CMB anisotropies observed today. Constraints on the physics responsible for the primodial power spectrum of these density fluctuations can be set with WMAP and $2 \mathrm{dF}$ data, under the assumption that the Hubble rate is dominated by the contribution from $\rho_{\phi}$ during the observable part of inflation [16]. The scalar and tensor perturbations with comoving wavenumber $k$ are solutions of the equation

$$
u_{k}^{\prime \prime}+\left(k^{2}-\frac{z^{\prime \prime}}{z}\right) u_{k}=0,
$$

where prime denotes derivative with respect to conformal time $\tau$, and $z=a \dot{\phi} / H$ for scalar perturbations while $z=a$ for the tensor case. The power spectra have the form

$$
\mathcal{P}_{\mathcal{R}}(k)=\frac{H^{2}}{\epsilon} f_{k} ; \quad \mathcal{P}_{h}(k)=H^{2} f_{k} ; \quad f_{k}=\frac{k^{3} u_{k}^{2}}{a^{2} H^{2}}
$$

where $\epsilon=-\dot{H} / H^{2}$. For standard inflation models 17], the solutions of (15) have the property that $u_{k}^{2} \rightarrow$ $a^{2} H^{2} / k^{3}$ for small wavenumbers $k / a H \rightarrow 0$, and thus $f_{k} \rightarrow$ constant. This gives the standard formulae for the spectral indices $n_{S}-1=-4 \epsilon-2 \delta$ and $n_{T}-1=-2 \epsilon$, where $\delta$ is defined by $\delta+\epsilon=\epsilon^{\prime} /(2 a H \epsilon)$.

In our model (15) can be solved in the limit $k / a H \rightarrow 0$ by changing variables from $\tau$ to $\theta$ :

$$
\begin{gathered}
\frac{d^{2} u_{k}}{d \theta^{2}}-\frac{2}{b}\left(1-3 \sin ^{2} \theta\right) \frac{d u_{k}}{d \theta}+c(\theta) u_{k}=0 \\
c(\theta)=\frac{6}{b} \cot \theta+1-\frac{8}{b^{2}}-\frac{6}{b^{2}}(\cos 2 \theta-1)-\frac{3}{b} \sin 2 \theta \\
c(\theta)=-\frac{8}{b^{2}}+\frac{12}{b^{2}} \sin ^{2} \theta
\end{gathered}
$$

where the first/second expression for $c(\theta)$ is for the scalar/tensor case. The solutions which match to the correct large $k$ behavior are:

$$
u_{k}=\frac{N}{\sqrt{k}} \frac{a}{k} \sin \theta ; \quad u_{k}=\frac{N}{\sqrt{k}} \frac{a}{k},
$$

for the scalar and tensor cases respectively, where $N$ is a constant.

We can plug these solutions into the expressions (16) for the power spectra, using also that, for our model, $\epsilon=3 \sin ^{2} \theta$. Remarkably, one finds that $n_{S}=n_{T}=1$.

To compare with contraints from primordial nucleosynthesis, we note that the second epoch of dark energy domination ended well before $a \simeq 10^{-10}$, the time at which nucleosynthesis occured. Indeed dark energy reheating effects are completely negligible from $a \simeq 10^{-10}$ until today. A possible exception is a small reheating effect occuring at very late times in our current epoch of accelerated expansion; this only occurs if the quintessence scalar is still managing to decay despite its incredibly small current mass of $\sim 10^{-36} \mathrm{eV}$. Even with such an effect, our model satisfies the constraints of successful nucleosynthesis [20].

Figure (2) also shows one (very brief) prior epoch of matter domination. This only occurs if there is some suitably heavy and long-lived matter around to go out of thermal equilibrium when $a \sim 10^{-22}$, e.g. a superheavy neutrino.

\section{REMARKS}

It is fair to say that, compared to the simple model presented above, the standard new inflation scenario looks rather extreme. In the evolution history portrayed in Figure 2 the interplay between inflationary expansion and reheating is much milder. In fact, apart from a few very brief periods, reheating effects in our model do not actually increase the temperature of the thermal radiation bath. Instead, the temperature is almost always decreasing, but it decreases more slowly than in the standard $\Lambda \mathrm{CDM}$ evolution.

Our particular realization of Slinky inflation must be regarded as a toy model (pun intended), since $\phi_{0}>M_{\mathrm{P}}$ and we have not invoked any consistent Planck scale framework such as string theory. However the model itself is surprisingly simple, and the physical picture which emerges from it has some compelling features. These are worthy of further investigation. Also, since the target space parametrized by our scalar is $\mathbf{S}^{\mathbf{1}}$, it would be interesting to extend this scenario to a class of nonlinear sigma models with other compact target spaces.

Fermilab is operated by Universities Research Association Inc. under Contract No. DE-AC02-76CH03000 with the U.S. Department of Energy. J.L. is grateful for the hospitality of José Bernabéu and the University of Valencia. We thank Scott Dodelson, Olga Mena, Chris Quigg, José Valle, and Jochen Weller for helpful discussions.

[1] S. Dodelson, M. Kaplinghat and E. Stewart, Phys. Rev. Lett. 85, 5276 (2000) [arXiv:astro-ph/0002360].

[2] P.J.E. Peebles \& B. Ratra, Phys. Rev. D37 3406 (1988); I. Zlatev, L. Wang, \& P.J. Steinhardt, Phys. Rev. Lett. 82, 896 (1998); P.J. Steinhardt, L. Wang, \& I. Zlatev, Phys. Rev. D59, 123504 (1999); A. Albrecht \& C. Skordis, astro-ph/9908085 (1999).

[3] P. J. E. Peebles and A. Vilenkin, Phys. Rev. D 59, 063505 (1999) [arXiv:astro-ph/9810509].

[4] M. Peloso and F. Rosati, JHEP 9912, 026 (1999) [arXiv:hep-ph/9908271].

[5] E. J. Copeland, A. R. Liddle and J. E. Lidsey, Phys. Rev. D 64, 023509 (2001) [arXiv:astro-ph/0006421].

[6] A. B. Kaganovich, Phys. Rev. D 63, 025022 (2001) [arXiv:hep-th/0007144]. 
[7] K. Dimopoulos and J. W. F. Valle, Astropart. Phys. 18, 287 (2002) [arXiv:astro-ph/0111417].

[8] A. S. Majumdar, Phys. Rev. D 64, 083503 (2001) [arXiv:astro-ph/0105518].

[9] G. Huey and J. E. Lidsey, Phys. Lett. B 514, 217 (2001) [arXiv:astro-ph/0104006].

[10] M. Sami and V. Sahni, Phys. Rev. D 70, 083513 (2004) [arXiv:hep-th/0402086].

[11] L. F. Abbott, E. Farhi and M. B. Wise, Phys. Lett. B 117, 29 (1982).

[12] G. Barenboim, O. Mena and C. Quigg, arXiv:astro$\mathrm{ph} / 0412010$.

[13] B. Feng, M. Li, Y. S. Piao and X. Zhang, arXiv:astro$\mathrm{ph} / 0407432$.

[14] S. M. Carroll, Phys. Rev. Lett. 81, 3067 (1998)
[arXiv:astro-ph/9806099].

[15] R. Fardon, A. E. Nelson and N. Weiner, JCAP 0410, 005 (2004) [arXiv:astro-ph/0309800].

[16] D. J. Schwarz and C. A. Terrero-Escalante, JCAP 0408, 003 (2004) [arXiv:hep-ph/0403129].

[17] E. D. Stewart and D. H. Lyth, Phys. Lett. B 302, 171 (1993) [arXiv:gr-qc/9302019].

[18] W. H. Kinney, E. W. Kolb, A. Melchiorri and A. Riotto, Phys. Rev. D 69, 103516 (2004) [arXiv:hep-ph/0305130].

[19] S. Eidelman et al. [Particle Data Group], Phys. Lett. B 592, 1 (2004).

[20] R. Bean, S. H. Hansen and A. Melchiorri, Nucl. Phys. Proc. Suppl. 110, 167 (2002) [arXiv:astro-ph/0201127]. 